\title{
Fabrication of Porous Scaffolds with a Controllable Microstructure and Mechanical Properties by Porogen Fusion Technique
}

\author{
Qinggang Tan ${ }^{1,2, *}$, Songgang $\mathrm{Li}^{3}$, Jie Ren ${ }^{1,2}$ and Chu Chen ${ }^{1}$
}

1 Institute of Nano- and Bio-Polymeric Materials, School of Materials Science and Engineering, Tongji University, 1239 Siping Road, Shanghai 200092, China; E-Mails: renjie65@163.com (J.R.); yourchenchu@163.com (C.C.)

2 Key Laboratory Advanced Civil Engineering Materials, Ministry of Education, School of Materials Science and Engineering, Tongji University, 1239 Siping Road, Shanghai 200092, China

3 Department of General Surgery, Xinhua Hospital, School of Medicine, Shanghai Jiaotong University, 1665 Kongjiang Road, Shanghai 200092, China; E-Mail: jxlisg@yahoo.cn

* Author to whom correspondence should be addressed; E-Mail: qgtan@ @ongji.edu.cn; Tel.: +86-21-69580234; Fax: +86-21-69580234.

Received: 2 November 2010; in revised form: 4 January 2011 / Accepted: 24 January 2011 / Published: 25 January 2011

\begin{abstract}
Macroporous scaffolds with controllable pore structure and mechanical properties were fabricated by a porogen fusion technique. Biodegradable material poly (D, L-lactide) (PDLLA) was used as the scaffold matrix. The effects of porogen size, PDLLA concentration and hydroxyapatite (HA) content on the scaffold morphology, porosity and mechanical properties were investigated. High porosity ( $90 \%$ and above) and highly interconnected structures were easily obtained and the pore size could be adjusted by varying the porogen size. With the increasing porogen size and PDLLA concentration, the porosity of scaffolds decreases, while its mechanical properties increase. The introduction of HA greatly increases the impact on pore structure, mechanical properties and water absorption ability of scaffolds, while it has comparatively little influence on its porosity under low HA contents. These results show that by adjusting processing parameters, scaffolds could afford a controllable pore size, exhibit suitable pore structure and high porosity, as well as good mechanical properties, and may serve as an excellent substrate for bone tissue engineering.
\end{abstract}


Keywords: tissue engineering; composite scaffolds; mechanical property; porogen fusion technique

\section{Introduction}

Tissue engineering aims to create medical devices that will repair, restore or regenerate tissues impaired by disease, injury, or age [1]. These devices are typically created by seeding a scaffold with cells. A scaffold has always been a key component in the tissue engineering field as it serves as the substrate for implanted cells and the physical support in forming new tissue [2,3]. Ideally, a tissue engineering scaffold needs to have adequate mechanical stability, mechanical strength and be of a highly open porous structure, in order that tissue can develop and tissue fluids and nutrients can transfer freely; pore size and its distribution should be suitable for cell in-growth [4-6]. The tissue engineering scaffold also needs to be easily fabricated into a variety of shapes and sizes. The materials for tissue engineering scaffolds should have controlled biodegradability or bioresorbability, so that scaffolds will eventually be replaced by the forming new tissue [7]. Therefore, the construction of scaffolds with controlled pore structures and mechanical properties is of crucial importance in the tissue engineering field.

In recent years, several techniques have been developed to fabricate tissue engineering scaffolds, including porogen leaching [8-10], gas expansion [11], emulsion freeze-drying [12], thermally induced phase separation [13-16] and 3D-printing [17], etc. Compared to other techniques, the porogen leaching technique provides easy control of pore structure and has been well established in the preparation of porous scaffolds for tissue engineering. This technique involves the casting of a polymer/porogen composite followed by aqueous washing out of the incorporated porogen. The pore size, porosity and pore morphology can be easily controlled by the properties of porogen. However, this technique may cause a problem of residual porogen in scaffolds, which may lead to poor pore interconnectivity and harm the cell seeding and culture. Moreover, only thin scaffold cross-sections can be produced due to the difficulty in removing salt particles deep in the matrix.

To avoid these disadvantages, a modified porogen leaching method was developed $[18,19]$. Only one procedure has proven viable: before the solvent casting process, the porogen fusion step is carried out under certain circumstances. This is called the porogen fusion technique. By use of this technique, a scaffold with desirable pore size, suitable porosity and pore morphology can easily be fabricated. The pore interconnectivity can also be greatly improved and the porogen residual problem is easily solved.

Biodegradable polyesters, such as poly (lactic acid) (PLA), poly (glycolic acid) (PGA) and their copolymers (PLGA), have been widely used for the preparation of tissue engineering scaffolds due to their good biodegradability and processing properties [20-22]. However, they are over flexible and of insufficient strength to meet the mechanical demands of bone replacement, nor do they present a favorable surface for cell attachment and proliferation because of their lack of specific cell-recognizable signals $[23,24]$.

As one of the primary components of extracellular bone matrix, apatite has high strength and exhibits unique advantages when used in bone reconstruction. Apatite also has good 
osteoconductivity [25], and high affinity to living cells [26]. Therefore, composites composed of synthetic biodegradable polymers and apatite, combine the bioactivity of bioactive apatite and the biodegradability of the polymer. These advantages cause an increasing interest in developing biodegradable polymer/apatite composites for repair, regeneration and tissue engineering of bone. Of all the composites, PLA or PLGA/calcium phosphate have been most extensively studied [9,14,26-28]. With the addition of calcium phosphate in composites, the mechanical strength is not only improved, but this can also lead to a more stable $\mathrm{pH}$ value than that of pure PLA or PLGA, due to the fact that the acidic degradation byproducts of PLA or PLGA are buffered by the calcium phosphate.

In this study, poly (D, L-lactide) (PDLLA) scaffolds with controllable structures were first fabricated by porogen fusion technique, and the effects of porogen size, polymer concentration on the microstructure and properties of scaffolds were investigated. Then, the (PDLLA)/hydroxyapatite (HA) composite scaffolds were prepared and the effect of HA on the structure and properties of composite scaffolds was also studied.

\section{Results and Discussion}

\subsection{Effect of Porogen Sizes on the Structure and Mechanical Properties of Scaffolds}

Figure 1. (A) Scanning Electron Microscopy (SEM) images of scaffolds with the same Biodegradable material poly (D, L-lactide) (PDLLA) concentration of $15 \%$ and different porogen sizes: (a) 200 300 $\mu \mathrm{m}$ (b) 300 450 $\mu \mathrm{m}$ (c) 450 900 $\mu \mathrm{m}$; (B) Scaffold sizes with the same PDLLA concentration of $15 \%$ and different porogen sizes calculated from the SEM images.

(A)

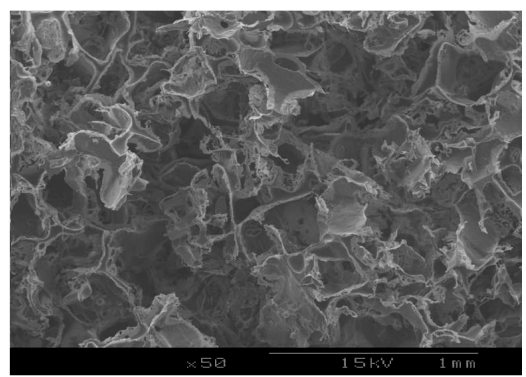

b

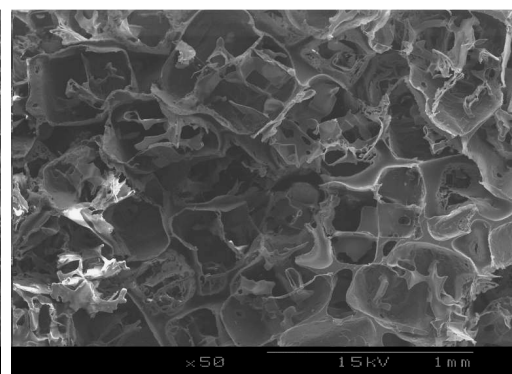

c

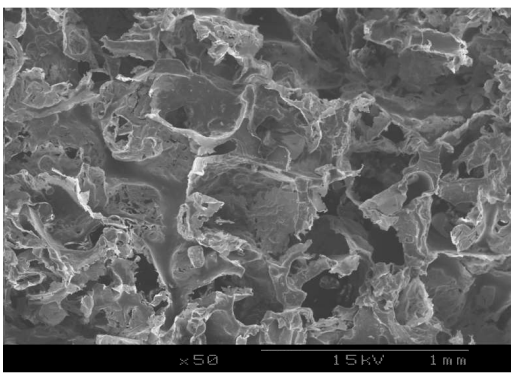

(B)

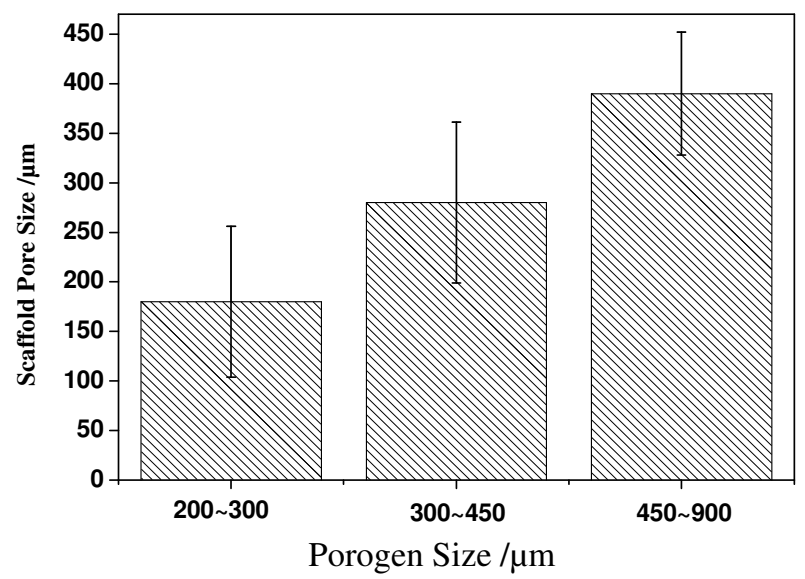


The effect of different porogen sizes on the structure of scaffolds was first studied by SEM. As is shown in Figure 1A, the microstructure of scaffolds varies with the different sizes of $\mathrm{NaCl}$ particles. Scaffolds fabricated using a porogen size of $200 \sim 300 \mu \mathrm{m}$, have irregular pore structures with a large number of holes in pore walls. The ones prepared using a porogen size of $300 \sim 450 \mu \mathrm{m}$ show comparatively regular pore structure, the pore walls become thicker and the number of holes in pore walls seems to slightly decrease. The scaffolds prepared with the largest porogen size of $450 \sim 900 \mu \mathrm{m}$ display the largest pore size, well-defined pore structure and very thick pore walls, but only very few intermittent holes in the pore walls, which may indicate that the interconnectivity of the scaffolds became poor. The pore size increases with the increasing sizes of the original $\mathrm{NaCl}$ particles (Figure 1B). However, the mean pore size is always smaller than that of $\mathrm{NaCl}$ particles. For example, the mean scaffold size with the porogen size of $300 \sim 450 \mu \mathrm{m}$ is only about $180 \mu \mathrm{m}$.

The macroporous polymer network was the replica of the surface of the bonded $\mathrm{NaCl}$ particles. In other words, the hollow space of the fused $\mathrm{NaCl}$ network formed the continuous skeleton of the scaffolds, and the pores were interconnected through the openings formed by the bonding areas of the $\mathrm{NaCl}$. The fusion technique successfully fused porogen together and resulted in the formation of holes in pore walls of scaffolds. However, the same fusion treatment to porogen particles with different sizes may lead to different results. When the porogen size is small, it is easier to moisten the particles and for them to fuse, which makes porogen shape inequable and leads to less hollow space in the fused porogen network. While larger porogen are not so easy to fuse, the shape of the porogen is better retained and the hollow space in the fused porogen networks is comparatively larger. More hollow space means that more polymer solution could be introduced to the scaffolds. On the other hand, when the size of the pore is smaller, the pore wall is subjected to a larger surface tension and this leads to more shrinkage. As a result, under the same fusion conditions, porogen with a smaller size resulted in disorganized pore structure and thinner pore walls; while porogen with a larger size resulted in well-defined pore structure and thicker pore walls. For similar reasons, this resulted in a substantial difference between the pore size and the size of $\mathrm{NaCl}$ particles.

Figure 2. The porosity of scaffolds with the same PDLLA concentration of $15 \mathrm{wt} \%$ and different porogen sizes.

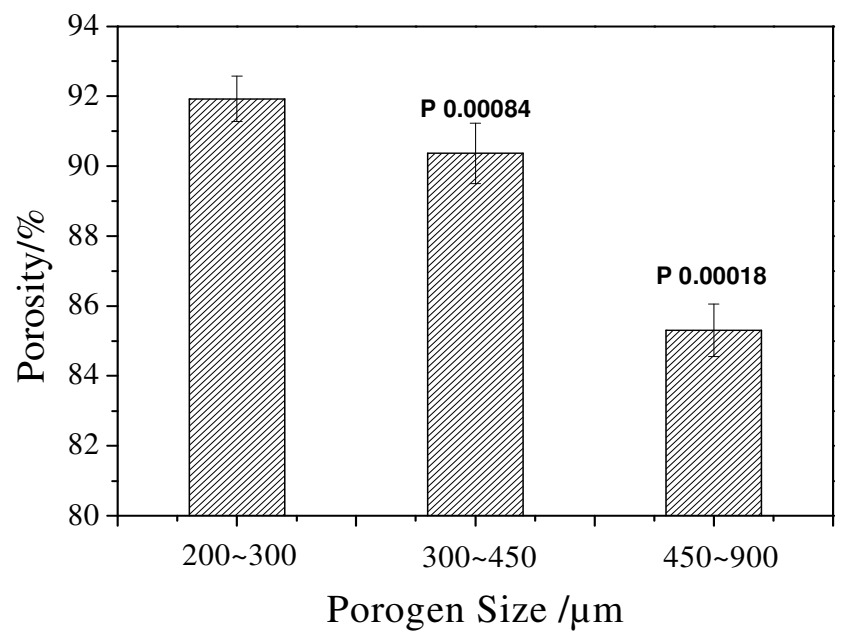


The effect of porogen with different sizes on the porosity of scaffolds is shown in Figure 2. It could be seen from the results that the porosity of scaffolds decreases with the increasing size of the porogen $(P<0.05)$. The results are consistent with the SEM analysis. When the size of porogen is small, the stacking density of porogen in a certain space is high compared to that of larger porogen. Moreover the porogen fusion treatment made the density higher; as a result, the space where the porogen was leached out became big. All these factors led to the result that porogen with small size creates a scaffold with high porosity.

The compressive strength of scaffolds tends to increase with the increasing size of porogen, as shown in Figure 3. When a porogen size of $200 \sim 300 \mu \mathrm{m}$ was used, the compressive strength of scaffolds is about $0.5 \mathrm{MPa}$; but when a porogen size of $450 \sim 900 \mu \mathrm{m}$ was used, the compressive strength reaches $1 \mathrm{MPa}(P<0.01$ compared with the size of $200 \sim 300 \mu \mathrm{m})$. But the change of compressive strength between $300 \sim 450 \mu \mathrm{m}$ and $450 \sim 900 \mu \mathrm{m}$ is not so obvious compared with that of between 200 300 $\mu \mathrm{m}$ and 300 450 $\mu \mathrm{m}$. Scaffolds prepared with porogen of a larger size have thicker pore walls and fewer small pores in the walls, which leads to the result that mechanical properties of scaffolds increase with the increasing size of porogen. When the small pores in the walls decrease, the effect of porogen size on the compressive strength also decreases, which led to the difference of compressive strength between 300 450 $\mu \mathrm{m}$ and 450 900 $\mu \mathrm{m}$ decrease. It is clear that the porogen size is a crucial parameter not only for controlling the pore structure, but also for adjusting the porosity and the mechanical properties of scaffolds.

However, these results are different from those found using sugar as porogen [18]. In this literature, using large sugar particles as porogen, the compressive strength, decreased with the increasing size of porogen. The difference in fusing ability and particle size between the salt particle and sugar particle may result in a different flowing behavior of the polymer solution and the pore structure of scaffolds. These factors perhaps led to the constrasting results.

Figure 3. The compressive strength of scaffolds with the same PDLLA concentration of $15 \mathrm{wt} \%$ and different porogen sizes.

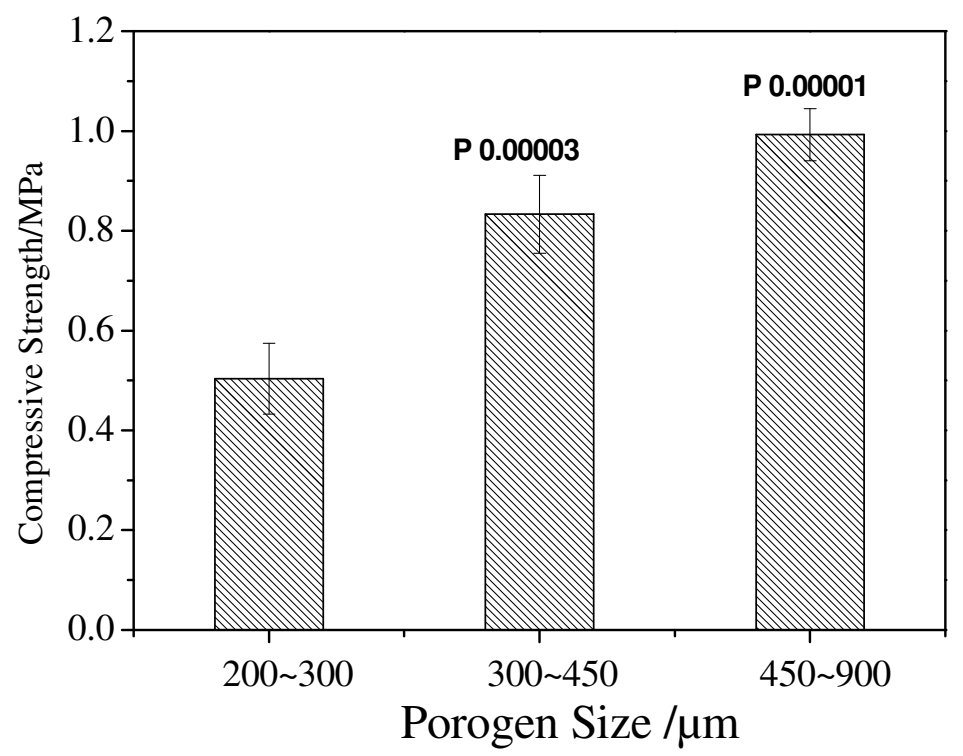




\subsection{Effect of PDLLA Concentration on the Structure and Mechanical Properties of Scaffolds}

Figure 4. (A) SEM images of scaffolds with the same porogen size of 300 450 $\mu \mathrm{m}$ and varying PDLLA concentrations: (a) $10 \mathrm{wt} \%$; (b) $12.5 \mathrm{wt} \%$; (c) $15 \mathrm{wt} \%$; (d) $17.5 \mathrm{wt} \%$; (e) $20 \mathrm{wt} \%$; (B) Scaffold sizes with the same porogen size of 300 450 $\mu \mathrm{m}$ and varying PDLLA concentrations calculated from SEM images.

(A)

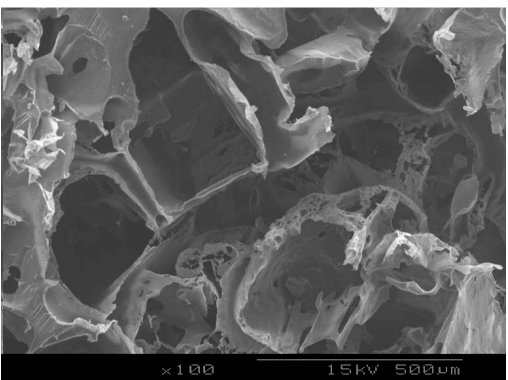

d

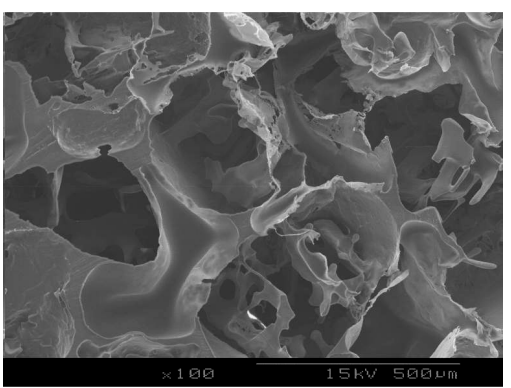

b

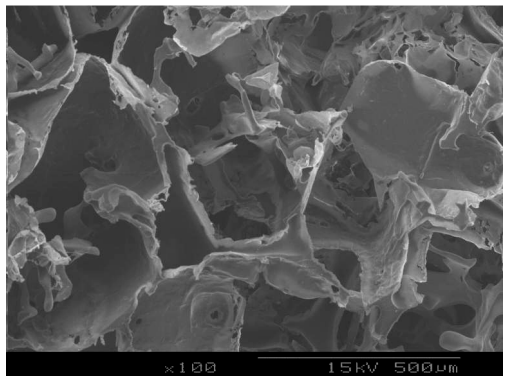

e

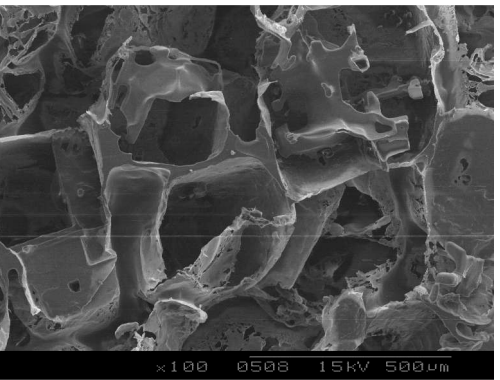

c

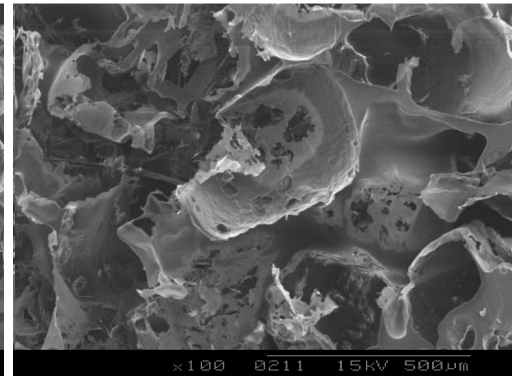

(B)

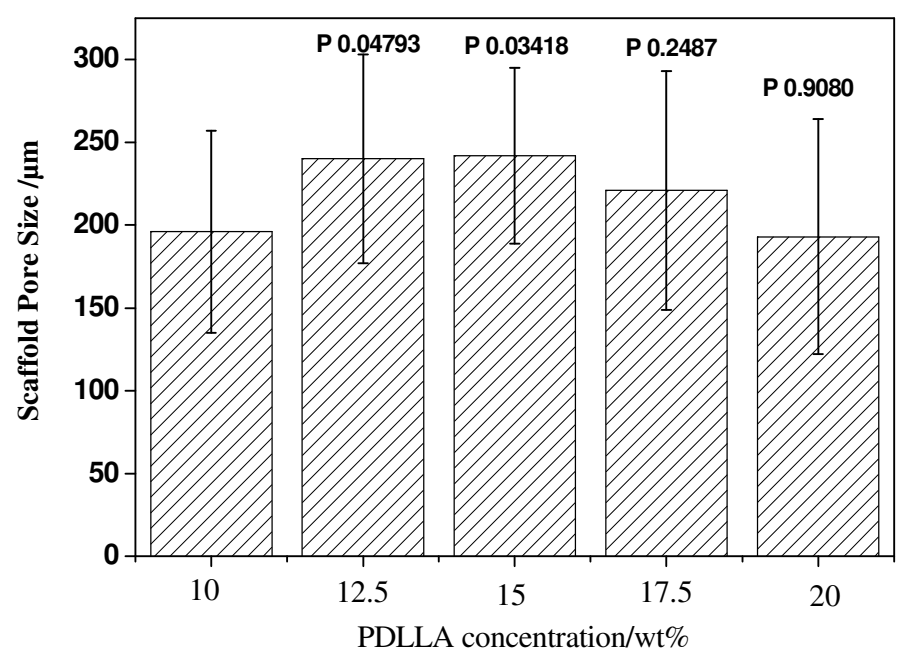

To study the effect of PDLLA concentrations on the properties of scaffolds, a series of scaffolds were prepared with the PDLLA concentration ranging from $10 \mathrm{wt} \%$ to $20 \mathrm{wt} \%$. SEM observation (Figure 4A) shows that the pore size of scaffolds prepared from a $10 \mathrm{wt} \%$ PDLLA solution is almost the same as that from the $17.5 \mathrm{wt} \%$ or $20 \mathrm{wt} \%$ PDLLA solution $(P>0.05)$; and the scaffold prepared from $12.5 \mathrm{wt} \%$ or $15 \mathrm{wt} \%$ has a slightly larger size $(P<0.01$ compared with that from the $10 \mathrm{wt} \%$ PDLLA solution as shown in Figure 4B). However, the scaffolds prepared from the $17.5 \mathrm{wt} \%$ or 
$20 \mathrm{wt} \%$ PDLLA solutions have a slightly different morphology from the scaffolds from $10 \mathrm{wt} \%$ or $12.5 \mathrm{wt} \%$ solutions, i.e., the scaffolds pore walls are thicker.

Figure 5. The porosity of scaffolds with the same porogen size 300 450 $\mu \mathrm{m}$ and different PDLLA concentrations.

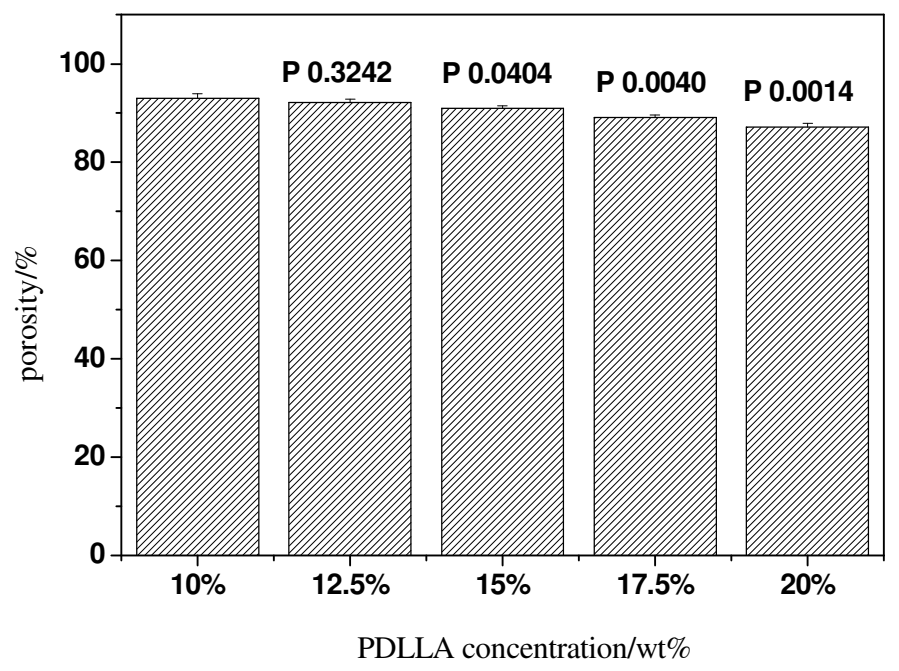

The thickness of the pore walls has certain effects on the porosity and mechanical properties of the scaffolds. The porosity and compressive strength test results show the porosity of scaffolds decreases from approximately $93 \%$ to $87 \%$ with increasing the concentration of PDLLA from $10 \mathrm{wt} \%$ to $20 \mathrm{wt} \%$, while its compressive strength exhibits a sharp increase from $0.4 \mathrm{MPa}$ to $1 \mathrm{MPa}$, as shown in Figure 5 and Figure 6.

Figure 6. The compressive strength of scaffolds with the same porogen size of 300 450 $\mu \mathrm{m}$ and different PDLLA concentrations.

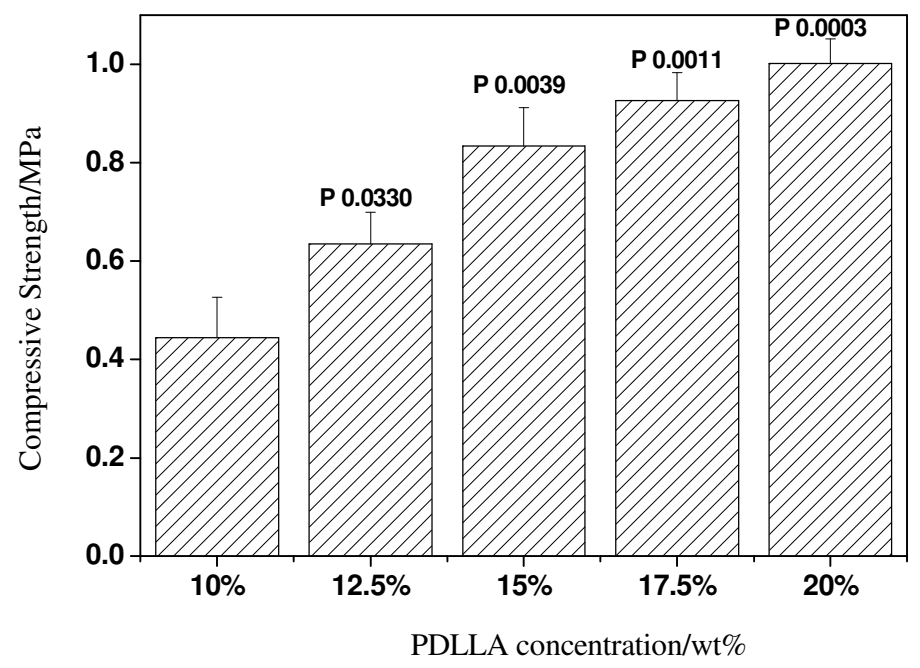

The polymer concentration or viscosity has a great effect on the porosity and compressive strength of scaffolds prepared with the fusion salt method [18]. For a defined space, a higher polymer concentration would have more polymers and produce an intact pore wall. These results led to the decreasing porosity and improved mechanical properties of scaffolds. A high polymer concentration 
also decreases the shrinkage of the pore size during the drying process. As a result, the pore size increased slightly as the PDLLA concentration increased. However, a too high polymer concentration would result in the solution having difficulty flowing into the fused salt scaffold. Consequently, the pore size slightly decreased at high PDLLA concentrations (17.5 wt $\%$ or $20 \mathrm{wt} \%$ ). The decrease in fluidity of the solution at high concentrations had no obvious effect on the porosity and compressive strength of scaffolds prepared from the $17.5 \mathrm{wt} \%$ PDLLA solution, compared with that of $20 \mathrm{wt} \%$ (the $P$ values are 0.0262 and 0.1612 , respectively.)

\subsection{Effect of HA Content on the Structure and Mechanical Properties of Scaffold}

Hydroxyapatite (HA) is the principal mineral component of natural bone. It has been extensively used for biomedical implant applications and bone regeneration due to its bioactive, biodegradable and osteoconductive properties. Incorporation of HA into a polymer matrix has often been carried out to increase osteoconductivity and biodegradability with significant enhancement of mechanical strength.

The effects of incorporating HA on the structure of scaffolds had also been investigated by varying the HA contents in the PDLLA solutions, while maintaining a constant PDLLA concentration. SEM observations show that the pore structure of scaffolds changes considerably with the varying HA contents (Figure 7). When there is no HA in scaffolds, the pore structure is not so regular and some membrane structures could be observed in scaffolds. But when HA is incorporated, the membrane structure disappears. Moreover, when increasing the HA content, the pore structure of scaffolds becomes more and more regular and the pore walls become thicker. This is presumably due to the high viscosity that HA brought into the PDLLA solution which enhanced the stability of the pore walls. The pore size also appeared to obviously increase when HA was incorporated into the scaffold (All $P$ values compared with no HA are less than 0.05 ). These results have demonstrated that the pore structure of the PDLLA scaffolds can be modified by the incorporation of HA.

Figure 7. (A) SEM images of scaffolds with the same porogen size $300 \sim 450 \mu \mathrm{m}$, the same PDLLA concentration $15 \mathrm{wt} \%$, and different HA contents: (a) $0 \mathrm{wt} \%$; (b) $5 \mathrm{wt} \%$; (c) $10 \mathrm{wt} \%$; (d) $15 \mathrm{wt} \%$ (e) $20 \mathrm{wt} \%$. (B) Scaffold sizes with the same porogen size 300 450 $\mu \mathrm{m}$, same PDLLA concentration $15 \mathrm{wt} \%$, and different HA contents calculated from the SEM images.

(A)

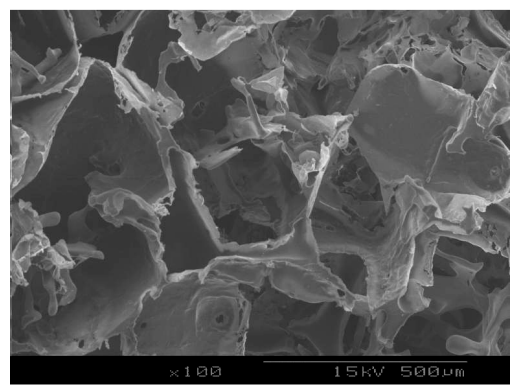

b

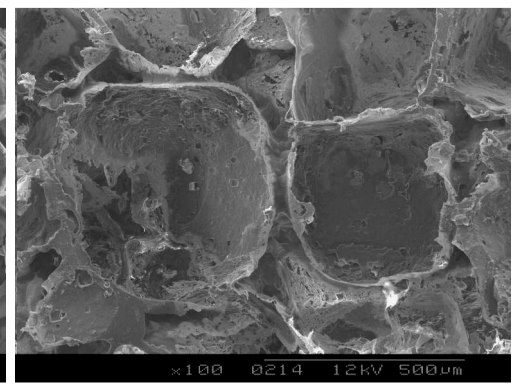

c

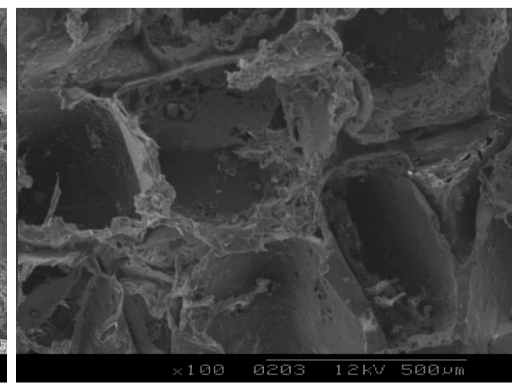


Figure 7. Cont.

(A)

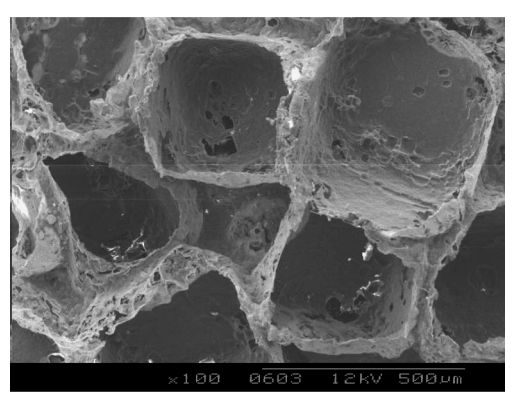

e

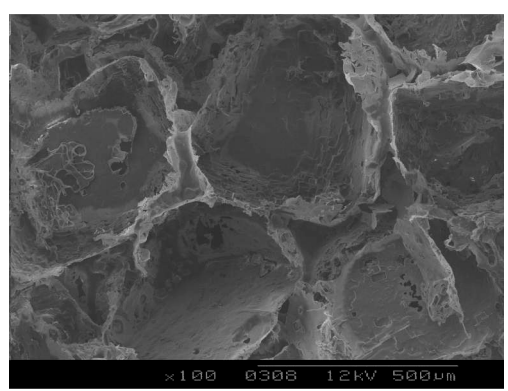

(B)

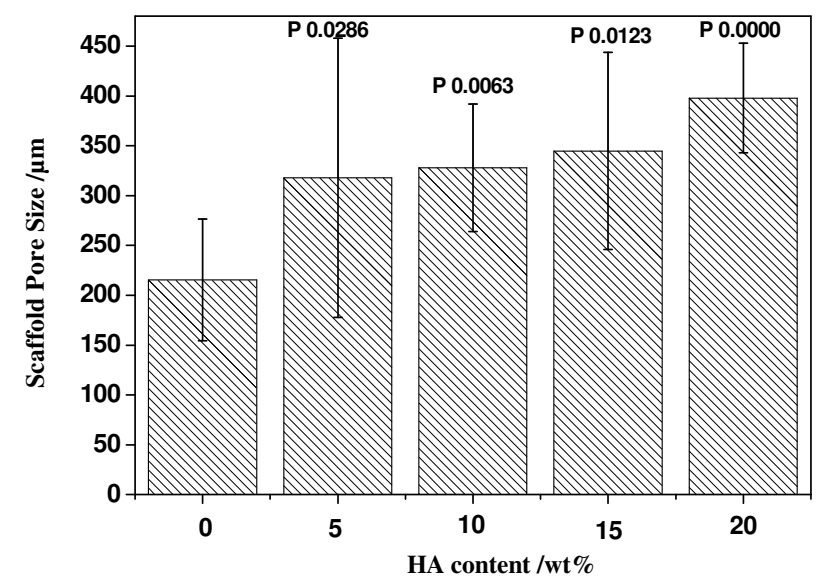

The content of HA also has some influence on the porosity of the PDLLA/HA composite scaffolds. Figure 8 shows that when the HA content is below $10 \mathrm{wt} \%$, the HA content has little impact on the porosity of the scaffolds, the porosities are all above 90\% (All $P$ values compared with no HA are larger than 0.05). But when the content of HA is over $10 \mathrm{wt} \%$, the porosity decreases (All $P$ values compared with no HA are less than 0.05). Large amounts of HA in the PDLLA solution might cause the agglomeration of the HA particles and block some small interconnected pores, which lowers the pore connectivity and decreases the porosity.

Figure 8. The porosity of the scaffolds with the same porogen size $300 \sim 450 \mu \mathrm{m}$, the same PDLLA concentration $15 \mathrm{wt} \%$, and different HA contents.

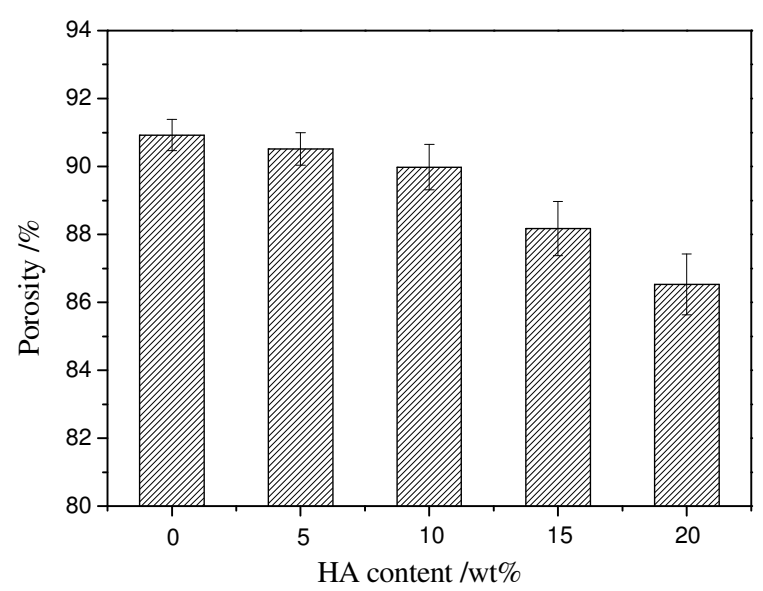


The incorporation of inorganic HA particles in scaffolds can perform as fillers to enhance the mechanical strength of the scaffolds. As seen from Figure 9, the compressive strength of scaffolds increases with the increasing HA content (All $P$ values compared with no HA are less than 0.05). With no HA in the scaffolds, the compressive strength of scaffolds was $0.8 \mathrm{MPa}$. When $5 \mathrm{wt} \% \mathrm{HA}$ is incorporated, the compressive strength is above $1.0 \mathrm{MPa}$, which is an increase of over $25 \%$. Even, when the HA content reaches $20 \mathrm{wt} \%$; the compressive strength was near $1.6 \mathrm{MPa}$, which is double the value of that of the original PDLLA scaffolds. These data demonstrate the positive effects of HA in enhancing the mechanical performance of scaffolds.

Figure 9. The compressive strength of scaffolds with the same porogen size of 300 450 $\mu \mathrm{m}$, same PDLLA concentration of $15 \mathrm{wt} \%$, and different HA contents.

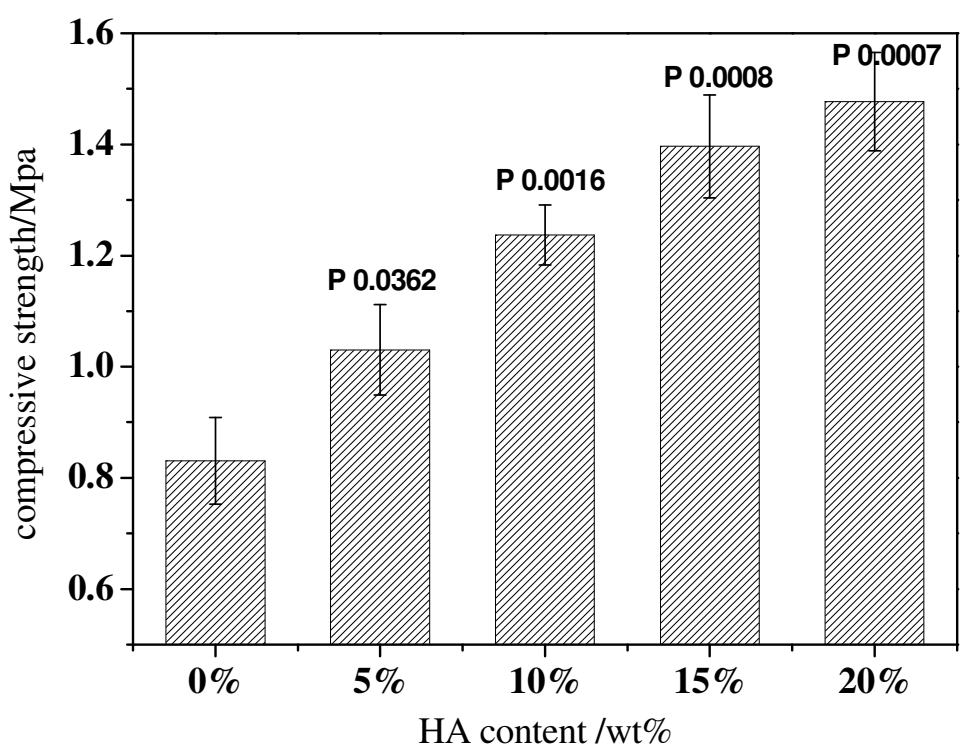

HA has good hydrophilic properties. Therefore, the introduction of HA in scaffolds would increase the water absorption of the composite scaffolds significantly, as presented in Figure 10. This is probably due to there being a large number of HA particles on the surface of scaffolds and therefore a large fraction of hydroxyl groups could be expected, which leads to an increase in water absorption. Although PDLLA matrix might not be helpful in cell seeding and culture, the incorporation of HA could not only greatly improve the mechanical properties and pore size but also help to enhance the hydrophilicity of the composite scaffolds, which might provide a favorable environment for cell ingrowths. 
Figure 10. Water absorption ability of PDLLA/HA scaffolds with different HA contents as a function of soaking time.

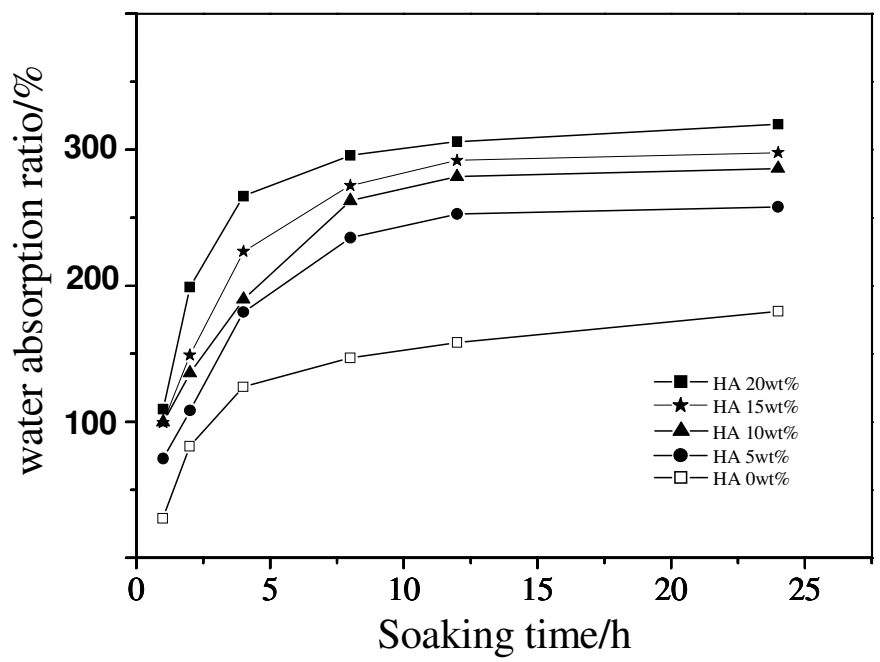

\section{Experimental Section}

\subsection{Materials}

The PDLLA ( $\mathrm{Mw}=200,000, \mathrm{PI}=2.3$ ) was synthesized using ring-opening polymerization. The HA nanoparticles, which were acicular crystals of about 100nm in length and 20 40 nm in width, was prepared by chemical precipitation method [23]. Chloroform and $\mathrm{NaCl}$ particles were purchased from the National Chemical Co. (China). The deionized water was twice distilled before use.

\subsection{Scaffold Preparation}

$\mathrm{NaCl}$ particles were seized at varying sizes: 200 300 $\mu \mathrm{m}, 300 \sim 450 \mu \mathrm{m}$ and $450 \sim 900 \mu \mathrm{m}$ respectively. The molds with diameter $16 \mathrm{~mm}$ and height $20 \mathrm{~mm}$ were shaped with aluminum foil. The molds were filled with $\mathrm{NaCl}$ particles of different size ranges and put in a humidified sealed container, which was placed in an incubator at $37^{\circ} \mathrm{C}$ for $10 \mathrm{~h}$. Afterwards the molds together with $\mathrm{NaCl}$ particles were dried at room temperature for at least $24 \mathrm{~h}$. Then $\mathrm{NaCl}$ particles formed a porous block.

After the formation of the porous block, PDLLA chloroform solution with a concentration of $10 \sim 20 \mathrm{wt} \%$ was poured into the molds, allowing the solution to diffuse throughout the mass of porogen. After $2 \sim 3 \mathrm{~min}$, the molds were transferred to a fridge at $-20{ }^{\circ} \mathrm{C}$ for $2 \mathrm{~h}$ to set the mixture. After the solvent was volatilized under room temperature for more than $48 \mathrm{~h}$, the PDLLA was precipitated and $\mathrm{NaCl}$ crystals were leached out of the precipitated PDLLA solution in deionized water at room temperature for 3 days. The deionized water was changed approximately 3 times each day. After dried in vacuum for $24 \mathrm{~h}$, PDLLA scaffolds were produced. The PDLLA/HA composite scaffolds were fabricated at the similar way as PDLLA scaffolds. The only difference was that HA was dispersed uniformly in the PDLLA solution before the solvent casting process.

To investigate the effect of the processing parameters on the scaffold properties, each group of scaffolds was prepared by only changing one processing parameter. 


\subsection{Characterization}

\subsubsection{Morphology}

The porous morphology of scaffolds was studied by scanning electron microscopy (SEM, Hitachi $\mathrm{S}-2360 \mathrm{~N})$. The specimens were cut with a razor blade after being frozen in $-50{ }^{\circ} \mathrm{C}$ conditions for $30 \mathrm{~min}$ and then they were coated with gold and observed by SEM at an accelerating voltage of $15 \mathrm{kV}$.

\subsubsection{Porosity}

The porosity of scaffolds was measured by liquid displacement method. Absolute ethanol with density $\rho$ was used as a displacement liquid because it can easily penetrate the scaffolds and would not induce shrinking or swelling as a non-solvent of the PDLLA. The specimens, pycnometer and ethanol were kept at $25{ }^{\circ} \mathrm{C}$ for $1 \mathrm{~h}$ before testing. A pycnometer filled with ethanol was weighed as $W_{1}$. A scaffold specimen of weight as $W_{\mathrm{S}}$ was immersed into the bottle, and then the bottle was submerged slowly until all the air in the scaffold was removed; lastly the pycnometer was refilled with ethanol and weighed as $W_{2}$. The scaffold saturated with ethanol was removed from the pycnometer and then the pycnometer was weighed as $W_{3}$.

The volume of the scaffold specimen is

$$
V_{\mathrm{S}}=\left(W_{1}-W_{2}+W_{\mathrm{S}}\right) / \rho
$$

while the total volume of the pores in it is

$$
V_{\mathrm{p}}=\left(W_{2}-W_{3}-W_{\mathrm{S}}\right) / \rho
$$

Then the porosity of the specimen $\varepsilon$ is

$$
\varepsilon=V_{\mathrm{p}} /\left(V_{\mathrm{p}}+V_{\mathrm{S}}\right)=\left(W_{2}-W_{3}-W_{\mathrm{S}}\right) /\left(W_{1}-W_{3}\right)
$$

\subsubsection{Compressive Strength}

The compressive strength of scaffolds was evaluated using a computer controlled DXLL-5000 mechanical tester. The top and bottom layers were removed from the original scaffolds to achieve flat surfaces. The testing was carried out according to the national test standards of P. R. China for compression of rigid cellular plastics (GB 8813-1988). Each specimen was made to be $16 \mathrm{~mm}$ in diameter and $3 \mathrm{~mm}$ thick. The testing was performed under the following conditions: dry/room temperature, a crosshead of $1 \mathrm{~mm} / \mathrm{min}$ was utilized. The compressive strength of scaffolds is defined as the stress when the specimen is compressed to $30 \%$ of its original thickness. Five specimens of each sample were tested.

\subsubsection{Water Absorption Ability}

The specimens with a dimension of $10 \mathrm{~mm} \times 10 \mathrm{~mm} \times 10 \mathrm{~mm}$ were dried and weighed as $W_{1}$, then immersed and soaked in twice-distilled water at room temperature $\left(25^{\circ} \mathrm{C}\right)$ for a given time, such as $1 \mathrm{~h}$, $2 \mathrm{~h}, 4 \mathrm{~h}, 8 \mathrm{~h}, 12 \mathrm{~h}$ and $24 \mathrm{~h}$. Subsequently, these specimens were taken out of the water; the excess 
water on the surface of the scaffold was carefully blotted with filter paper and then weighed as $\mathrm{W}_{2}$. The water absorption ratio $\alpha$ for specimens at different time is defined as:

$$
\alpha=\left(W_{2}-W_{1}\right) / W_{1} \times 100 \%
$$

\section{Conclusions}

Biodegradable porous scaffolds were prepared by porogen fusion technique. The effects of porogen size, PDLLA concentration and HA content on the microstructure and properties of the scaffolds were studied. Interconnected open pore structures with small pores in the pore walls are observed. The pore size can be easily controlled by adjusting the porogen size. With the increase of porogen size, PDLLA concentration and HA content, the porosity of scaffolds decreases while the mechanical properties increase. The incorporation of HA greatly improves the pore size, mechanical properties and the hydrophilicity while maintaining the porosity under $10 \%$ of HA content in scaffold. The SEM, porosity and compressive strength test results indicate that the microstructure and mechanical properties of scaffolds could be precisely controlled by adjusting the processing parameters.

In conclusion, the microstructure and properties of the scaffolds can be well controlled using porogen fusion technique and the scaffolds may serve as a good substrate in the bone tissue engineering field.

\section{Acknowledgements}

The project was sponsored by the national science foundation of china (NSFC-50603019), Nano Science and Technology Special Funding of Shanghai Science and Technology Commission (Grant number: 0952nm03600, 1052nm05400) and Key Basic Research Project from Shanghai Science and Technology Commission (Grant number: 08JC1416200).

\section{References}

1. Hutmacher, D.W. Scaffolds in tissue engineering bone and cartilage. Biomaterials 2000, 21, 2529-2543.

2. Temenoff, J.S.; Mikos, A.G. Tissue engineering for regeneration of articular cartilage. Biomaterials 2000, 21, 431-440.

3. Zhang, Y.; Zhang, M. Synthesis and characterization of macroporous chitosan/calcium phosphate composite scaffolds for tissue engineering. J. Biomed. Mater. Res. 2001, 55, 304-312.

4. Rizzi, S.C.; Heath, D.J.; Coombes, A.G.A.; Bock, N.; Textor, M.; Downes, S. Biodegradable polymer/hydroxyapatite composites: Surface analysis and initial attachment of human osteoblasts. J. Biomed. Mater. Res. 2001, 55, 475-486.

5. Wang, M. Developing bioactive composite materials for tissue replacement. Biomaterials 2003, 24, 2133-2151.

6. Karageorgiou, V.; Kaplan, D. Porosity of 3D biomaterial scaffolds and osteogenesis. Biomaterials 2005, 26, 5474-5491. 
7. Widmer, M.S.; Puneet, K.; Gupta, P.K.; Lu, L.; Meszlenyi, R.K.; Evans G.R.D., Brandt, K.; Savel, T.; Gurlek, A.; Charles, W.; Patrick, C.W.; Mikos, A.G. Manufacture of porous biodegradable polymer conduits by an extrusion process for guided tissue regeneration. Biomaterials 1998, 19, 1945-1955.

8. Mikos, A.G.; Lyman, M.D.; Freed, L.E.; Langer, R. Wetting of poly(L-lactic acid) and poly(D,L-lactic-co-glycolic acid) foams for tissue culture. Biomaterials 1994, 15, 55-58.

9. Kim, S.S.; Park, M.S.; Jeon, O.; Choi, C.Y.; Kim, B.S. Poly(lactide-co-glycolide)/hydroxyapatite composite scaffolds for bone tissue engineering. Biomaterials 2006, 27, 1399-1409.

10. Chen, G.; Ushida, T.; Tateishi, T. Preparation of poly(L-lactic acid) and poly(DL-lactic-co-glycolic acid) foams by use of ice microparticulates. Biomaterials 2001, 22, 2563-2567.

11. Mooney, D.J.; Baldwin, D.F.; Suh, N.P.; Vacanti, J.P.; Langer, R. Novel approach to fabricate porous sponges of poly (DL-lactic-co-glycolic acid) without the use of organic solvents. Biomaterials 1996, 17, 1417-1422.

12. Wang, K.; Thomas, C.H.; Healy, K.E.; Nuber, G. A novel method to fabricate bioabsorbable scaffolds. Polymer 1995, 36, 837-842.

13. Nam, Y.S.; Park, T.G. Biodegradable polymeric microcellular foams by modified thermally induced phase separation method. Biomaterials 1999, 20, 1783-1790.

14. Zhang, R.; Ma, P.X. Poly( $\alpha$-hydroxyl acids)/hydroxyapatite porous composites for bone-tissue engineering. I. Preparation and morphology. J. Biomed. Mater. Res. 1999, 44, 446-455.

15. Hua, F.J.; Park, T.G., Lee, D.S. A facile preparation of highly interconnected macroporous poly (D,L-lactic acid-co-glycolic acid) (PLGA) scaffold by liquid-liquid phase separation of a PLGA-dioxane-water ternary system. Polymer 2003, 44, 1911-1920.

16. Hua, F.J.; Nam, J.D.; Lee, D.S. Preparation of a macroporous poly(L-lactide) scaffold by liquid-liquid phase separation of a PLLA/1,4-dioxane/water ternary system in the presence of NaCl. Macromol. Rapid Commun. 2001, 22, 1053-1057.

17. Hutmacher, D.W. Scaffold design and fabrication technologies for engineering tissuesstate of the art and future perspectives. J. Biomater. Sci. Polym. Ed. 2001, 1, 107-124.

18. Guan, L.; Davies, J.E. Preparation and characterization of a highly macroporous biodegradable composite tissue engineering scaffold. J. Biomed. Mater. Res. 2004, 71A, 480-487.

19. Murphy, W.L.; Dennis, R.G.; Kileny, J.L.; Mooney, D.J. Salt fusion: an approach to improve pore interconnectivity within tissue engineering scaffolds. Tiss. Eng. 2002, 8, 43-52.

20. Lu, L.C.; Garcia, C.A.; Mikos, A.G. In vitro degradation of thin poly (DL-lactic-co-glycolic acid) films. J. Biomed. Mater. Res. 1999, 46, 236-244.

21. Migliaresi, C.; Fambri, L.; Cohn, D. A study on the in vitro degradation of poly (lactic acid). J. Biomed. Sci. Polym. Ed. 1994, 6, 591-606.

22. Ge, Z.; Yang, F.; Goh, J.C.H.; Ramakrishna, S.; Lee, E.H. Biomaterial and scaffolds for ligament tissue engineering. J. Biomed. Mater. Res. 2006, 77A, 639-652.

23. Barrera, D.A.; Zylstra, E.; Lansbury, P.T.; Langer, R. Synthesis and RGD peptide modification of a new biodegradable copolymer: poly (lactic acid-co-lysine). J. Am. Chem. Soc. 1993, 115, 11010-11011. 
24. Suh, H.; Hwang, Y.S.; Lee, J.E.; Han, C.D.; Park, J.C. Behavior of osteoblasts on a type I atelocollagen grafted ozone oxidized poly-L-lactic acid membrane. Biomaterials 2001, 22, 219-230.

25. Hench, L.L. Bioceramics: From concept to clinic. J. Am. Ceram. Soc. 1991, 74, 1487-1510.

26. Rizzi, S.C.; Heath, D.J.; Coombes, A.G.A.; Bock, N.; Textor, M.; Downes, S. Biodegradable polymer/hydroxyapatite composites: Surface analysis and initial attachment of human osteoblasts. J. Biomed. Mater. Res. 2001, 55, 475-486.

27. Bleach, N.C.; Nazhat, S.N.; Tanner, K.E.; Kellomaki, M.; Tormala, P. Effect of filler content on mechanical and dynamic mechanical properties of particulate biphasic calcium phosphate-polylactide composites. Biomaterials 2002, 7, 1579-1585.

28. Montse, C.H.; Sergio, D.V.; Hentges, E.; Bleuet, P.; Lacroix, D.; Planell, J.A. Mechanical and structural characterisation of completely degradable polylactic acid/calcium phosphate glass scaffolds. Biomaterials 2007, 30, 4429-4438.

(C) 2011 by the authors; licensee MDPI, Basel, Switzerland. This article is an open access article distributed under the terms and conditions of the Creative Commons Attribution license (http://creativecommons.org/licenses/by/3.0/). 